\title{
A new composition-dependent equation of state for clinopyroxene
}

\author{
S. SCHORN ${ }^{1} \&$ E.C.R. GREEN ${ }^{1}$
}

${ }^{1}$ School of Earth Sciences, University of Melbourne, Parkville, Victoria 3010, Australia (simon.schorn@unimelb.edu.au)

Thermodynamic modelling has become a widely employed tool in petrology, geochemistry and geodynamics. Such modelling requires realistic composition-dependent equations of state $(x$-eos) for minerals of petrological interest. Clinopyroxene (cpx) is a common phase in the Earth's mantle and mafic- to intermediate lithologies. There are currently several calibrated $x$-eos for cpx, available for calculations in phase equilibrium software packages such as Perple_X and THERMOCALC. The various $x$-eos are calibrated in different regions of pressuretemperature-composition $\quad(P-T-\mathbf{x})$ space. However, these $x$-eos are not thermodynamically consistent with each other, and therefore cannot be used in the forward modelling of processes that encompass more than one of these regimes.

We are developing a single $x$-eos to represent cpx in common crustal and uppermost-mantle environments, built on the Holland \& Powell (2011) dataset [1]. The first step was to amalgamate, as far as possible, the structures and parameterisations of the existing $x$-eos for omphacitic cpx [2], low-pressure, low-Na suprasolidus cpx with significant tetrahedral $\mathrm{Al}$ [3] and mantle cpx at $<50$ kbar [4]. Next, we must reconcile their inconsistencies by recalibrating against the data originally used in their calibration. Further, we have added high-pressure, lowtemperature data (e.g. [5]) to this database that was not used in previous calibrations. The resulting $x$-eos will be suitable for large-scale integrated thermodynamic/geodynamic simulations spanning a wide range of crustal and upper mantle conditions and compositions.

[1] Holland \& Powell (2011) J Metam Geol 29, 333-383. [2] Green et al. (2007) Am Min 92, 1181-1189. [3] Green et al. (2016) J Metam Geol 34, 845-869. [4] Holland et al. (2018) J Petrol 59, 881-900. [5] Poli (1993) Am J Sci 293, 1061-1107. 